\title{
Estrategias de aprendizaje, comprensión lectora y rendimiento académico en Educación Secundaria
}

\author{
Natalia Solano Pinto \\ Universidad Castilla La Mancha - Toledo - España \\ Ana Isabel Manzanal Martínez \\ Universidad Internacional de La Rioja - Madrid - España \\ Lourdes Jiménez-Taracido \\ Universidad Internacional de La Rioja - Madrid - España
}

\begin{abstract}
Resumen
En este artículo se persigue analizar las estrategias de aprendizaje, el control de la compresión lectora y el rendimiento académico, en Lengua castellana y Matemáticas, para una muestra de 118 alumnos de Educación Secundaria en Madrid. Los resultados evidenciaron diferencias significativas entre el grupo clasificado como lector hábil y el no hábil. De hecho, en el grupo lector hábil predominan el control emocional $(F=$ 8.52, $p=.004)$, la selección de información relevante $(F=12.17, p=.001)$, el pensamiento crítico y creativo $(F=6.06, p=.015)$, la recuperación de la información aprendida $(F=5.55, p=.014)$ y la planificación y la evaluación de la información como parte del proceso de metacognición ( $F$ $=3.64, p=.014)$. Por último, existen indicios de que el control de la comprensión lectora pueda explicar en parte el rendimiento académico y por tanto ser una variable predictora.
\end{abstract}

Palabras clave: Aprendizaje; lectura; rendimiento escolar.

\section{Learning strategies, reading comprehension and academic achievement in}

\section{Secondary Education}

\begin{abstract}
This paper analyses learning strategies and reading comprehension control with academic achievement in the subjects of Language and Mathematics in 118 students from Secondary Education in Madrid. The results showed significative differences between skilled reader / non skilled reader groups. In fact, in skilled reader group prevail emotional control $(F=8.52, p=.004)$, selection of relevant information $(F=12.17, p=.001)$, critical and creative thinking $(F=6.06, p=.015)$, recovery of learnt information $(F=5.55, p=.014)$ and planification and evaluation for results as a part of metacognitive processes. Finally, reading comprehension control could explain partially academic performance in these subjects and so it could be a predictor variable.
\end{abstract}

Keywords: Learning; reading; academic achievement.

\section{Estratégias de aprendizagem, compreensão leitora e rendimento acadêmico na Educação Secundária}

\section{Resumo}

Neste artigo busca-se analisar as estratégias de aprendizagem, o controle da compreensão leitora e o rendimento acadêmico em Língua castelhana e Matemática em uma amostra de 118 alunos de Educação Secundária de Madri. Os resultados evidenciaram diferenças significativas entre os grupos classificados como leitor hábil e não hábil. De fato, no grupo leitor hábil predominam o controle emocional $(F=8.52, p=.004)$, a seleção de informação relevante $(F=12.17, p=.001)$, o pensamento crítico e criativo $(F=6.06, p=.015)$, a recuperação da informação aprendida $(F$ $=5.55, p=.014)$ e o planejamento e a avaliação da informação como parte do processo de metacognição $(F=3.64, p=.014)$. Por último, há indícios de que o controle da compreensão leitora possa explicar em parte o rendimento acadêmico e portanto ser uma variável preditora.

Palavras-chave: Aprendizagem; leitura; rendimento escolar. 


\section{Introducción}

El aprendizaje autorregulado (SRL en sus siglas en inglés: Self-regulated learning) se ha asentado en las últimas décadas como uno de los elementos clave del aprendizaje (Zimmerman, 2011; Azevedo \& Aleven, 2013). Implica que el sujeto controla y planifica las actividades mentales que lleva a cabo, y tal es su importancia, que numerosos autores estiman que éste puede ayudar a predecir el éxito académico (Dignath \& Büttner, 2008; Sitzmann \& Ely, 2011). Según el SRL, para que se logre un aprendizaje significativo es necesario la activación de las estrategias adecuadas en cada uno de los procesos implicados en la adquisición del conocimiento (Solano, González-Pienda, González-Pumariega, \& Núñez, 2004; González-Pienda, Fernández, Bernardo, Núñez, \&Rosário, 2014).

El Programa Internacional para la Evaluación de Estudiantes (PISA) evalúa el rendimiento del alumnado en tres aspectos: comprensión lectora; matemáticas y resolución de problemas; y comprensión de textos científicos. Según los datos del informe PISA, el rendimiento académico del alumnado español es superado por la mayoría de los países de la Unión Europea. Los resultados manifiestan un bajo nivel de comprensión lectora al término de la educación obligatoria en países como España (González, Barba, \& González, 2010), México (Madero \& Gómez, 2013) y Brasil (Mezzalira \& Boruchovitch, 2014), entre otros. Por tanto, la comprensión lectora es uno de estos procesos que han cobrado mayor relevancia a raíz de los resultados de las pruebas estandarizadas PISA (INEE, 2013). En este sentido, algunos autores señalan que entre las causas que podrían explicar estos resultados se encuentra la deficiencia en la producción y aplicación de estrategias de comprensión lectora (González \& cols., 2010).

En el marco del SRL, las estrategias de aprendizaje, y dentro de ellas las de lectura, se organizan en tres categorías en función del tipo de proceso de pensamiento: las estrategias cognitivas, metacognitivas y afectivas-sociales (Anastasious \& Griva, 2009; Gutiérrez-Braojo, Rodríguez, \& Salmerón-Vílchez, 2014). Las primeras abarcan procesos de organización, transformación, elaboración, memorización, práctica o transferencia de información, las segundas incluyen el monitoreo activo (evaluación), la regulación y planificación del propio proceso cognitivo (Taylor \& Hamm, 2016). Por último, las estrategias motivacionales, relativas a los factores de expectativas, valor y afectividad, suscitan cogniciones y emociones en el alumno con respecto a las acciones durante la ejecución, y por tanto, inciden en las estrategias cognitivas y metacognitivas a desarrollar (Mezzalira \& Boruchovitch, 2014). Además, se considera que en todos los niveles educativos (primaria, secundaria y superior) es fundamental la autorregulación, ya que se piensa que es un proceso responsable de la activación de las estrategias de aprendizaje y por lo tanto, es un factor predictor del rendimiento escolar (Dignath \& Büttner, 2008; Sitzmann \& Ely, 2011).

El estudio conjunto de los tres tipos de estrategias sobre el proceso de comprensión lectora no es muy frecuente en la literatura. En este sentido, Zimmerman (2011) señala la necesidad de desarrollar modelos integradores que se ajusten a la realidad, ya que el alumno puede regular proactivamente su cognición, motivación y comportamiento con el fin de cumplir determinados objetivos y así mejorar su rendimiento académico. Dicha necesidad se viene reflejando en el aumento de las investigaciones sobre los aspectos cognitivos, metacognitivos, motivacionales y emocionales implicados en las competencias lectoras de los estudiantes (Cano, García, Justicia, \& García-Berbén, 2014; Bernacky, Nokes-Malch, \& Aleven, 2015; Boudreaux, 2016; Turkyilmaz, 2016).

Con el objetivo de poner a prueba los modelos teóricos que relacionan distintas variables con el rendimiento académico, Miñano y Castejón (2011) realizaron un modelo estructural. El estudio se realizó con una muestra de 369 estudiantes de la ESO. Las distintas variables evaluadas fueron: aptitudes en Lengua castellana y Matemáticas con una Batería de Aptitudes Generales y Diferenciales (Yuste, Martínez, \& Galve, 2005, conforme citado por Miñano \& Castejón, 2011); autoconcepto académico evaluado con la Escala de Evaluación de Autoconcepto para adolescentes (González-Pienda, 2002, conforme citado por Miñano \& Castejón, 2011); Orientaciones de meta y Atribuciones causales medido con el cuestionario de Motivación hacia el Aprendizaje y la escala de estilos atributivos (Alonso \& Sánchez, 2002 conforme citado por Miñano \& Castejón, 2011); rendimiento académico obtenido a partir de las calificaciones del alumnado y las estrategias de aprendizaje evaluado con las subescalas cognitivas y metacognitivas del Cuestionario de Aprendizaje (CEA, Beltrán, Pérez, \& Ortega, 2006). Entre los resultados obtenidos destacan que las estrategias de aprendizaje tienen un papel mediador en el rendimiento académico, destacando un efecto directo con aptitud verbal $(\beta=.193 ; p<.000)$, calificaciones en Lengua castellana $(\beta=$ $.098 ; p<.000)$ y con la aptitud matemática $(\beta=.213 ; p<.000)$ y calificaciones de Matemáticas ( $\beta=.087 ; \beta<.000$ ).

Por otro lado, en función de las estrategias empleadas durante la comprensión es posible determinar un perfil de los estudiantes. Así, los considerados buenos lectores detectan más errores mientras realizan la lectura (Tausch, 2012), utilizan estrategias adecuadas en función de la demanda de la lectura (Horner \& Shwery, 2002), usan más frecuentemente estrategias metacognitivas de planificación y control de la comprensión y cognitivas para la elaboración de las tareas (Yang, 2012) y manifiestan creencias positivas en torno a la lectura y a sí mismos como lectores (Madero \& Gómez, 2013). En este sentido, Dermitzaki, Andreou, y Paraskeva (2008) analizaron las diferencias entre los grupos denominados altos y bajos en comprensión lectora según las estrategias conductuales y la propia ejecución en la situación de comprensión lectora. Los alumnos que mostraron una adecuada comprensión lectora destacan en estrategias como auto-activación, persistencia ante dificultades y automotivación.

Es indispensable el papel del control de la comprensión durante la lectura y de las creencias positivas hacia este proceso, pues actúan como precursoras del pensamiento 
metacognitivo (Madero \& Gómez, 2013). A falta de éste, los lectores no detectan problemas durante el proceso de comprensión socavando la posibilidad de solventarlos. Sin embargo, la situación que frecuentemente se reporta es que la mayoría de los sujetos poseen una escasa conciencia del nivel real de comprensión, demostrando falta de dominio tanto en las estrategias de evaluación como en la regulación de su propia comprensión (Mazzitelli, Maturano, \& Macías, 2007; Jiménez-Taracido, Baridón, \& Manzanal, 2016).

En este contexto resulta imprescindible investigar la interacción de variables como la metacognición y el efecto sobre la autorregulación del proceso de aprendizaje (Efklides, 2011), sobre el comportamiento lector (Ramirez, Rossel, \& Nazar, 2015) y sobre el rendimiento académico (Young \& Fry, 2008).

El objetivo principal del presente estudio persigue indagar sobre las interrelaciones entre las siguientes variables: control de la comprensión lectora, estrategias de aprendizaje y rendimiento académico. Para ello se procede a caracterizar una muestra de alumnos de educación secundaria en base a su habilidad lectora con textos académicos, sobre las estrategias de aprendizaje que habitualmente usan y las calificaciones obtenidas en asignaturas de Lengua castellana y Matemáticas. Finalmente, se persigue valorar si existe un modelo predictivo del control de la comprensión lectora en relación al rendimiento académico

\section{Método}

\section{Participantes}

La muestra estuvo formada por 118 estudiantes de $1^{\circ}$ curso de Educación Secundaria Obligatoria (ESO) procedentes de dos centros educativos de titularidad privada-concertada de la Comunidad de Madrid (España). Todos los sujetos tenían edades entre 12 -13 años y su distribución corresponde a $48,8 \%$ de mujeres y $51,2 \%$ de hombres. La obtención de la muestra fue de conveniencia. Los datos se obtuvieron en el año 2013. Se solicitó el consentimiento informado a las familias de los participantes.

\section{Instrumentos y medidas}

Se evaluaron tres variables: Rendimiento académico, estrategias de aprendizaje y control de la comprensión lectora. A continuación se pasa a comentar los instrumentos empleados para obtener las medidas de las mencionadas variables:

- Rendimiento académico: se solicitó a los dos centros educativos de la muestra las calificaciones del alumnado obtenidas en relación a las asignaturas de Lengua castellana y Matemáticas.

- Estrategias de aprendizaje: El instrumento elegido fue el cuestionario CEA elaborado por Beltrán y cols. (2006), que muestra adecuadas propiedades psicométricas en los estudios previos, cuyos datos están disponibles en el manual publicado por los autores.

El cuestionario consta de 70 ítems y tiene un coeficiente de fiabilidad de alfa para el total de la prueba de .95 Está configurado por cuatro escalas, que a continuación se describen:

1. Metacognición: Se divide en dos subescalas: planificación y evaluación, y regulación. Consta de 11 ítems (7 para planificación y evaluación y 4 para regulación). El coeficiente de fiabilidad de alfa es de .77 .

2. Sensibilización: dividido en tres subescalas: motivación (12 ítems); actitudes (3 ítems) y afectividad-control emocional (5 ítems). La escala de sensibilización consta de 20 ítems y el coeficiente de fiabilidad de alfa es de .82 .

3. Elaboración: compuesta por tres subescalas: selección de la información (4 ítems); organización de la información (4 ítems); y elaboración de la información (9 ítems). La escala de elaboración consta de 17 ítems y el coeficiente de fiabilidad de alfa es de .87 .

4. Personalización: Esta escala se divide en tres subescalas: pensamiento crítico y creativo (10 ítems- 7 para pensamiento crítico y 3 para creativo-); subescala de recuperación (4 ítems); y subescala de transferencia (7 ítems). La escala de personalización consta de 22 ítems y su coeficiente de fiabilidad de alfa es de .88 .

- Control de la comprensión lectora (CCL, en adelante): se aplicó un instrumento construido ad hoc que permite medir el control de la comprensión de los estudiantes por medio de textos científicos cortos con inconsistencias (Jiménez-Taracido \& cols., 2016). Dicho instrumento corresponde a una adaptación del creado por Otero y Campanario (1990) y utilizado en sus investigaciones (Otero, Campanario, \& Hopkins, 1992; García-Arista \& cols., 2006), basado en la detección de contradicciones explícitas, con el fin de averiguar si el lector comprende los textos y su evaluación metacognitiva.

El alfa de Cronbach calculado para el instrumento en esta muestra es de .928, lo cual indica un nivel alto de consistencia interna de la prueba y con ello su fiabilidad para medir el CCL de los estudiantes participantes. También se determinó el grado de concordancia inter-observador entre la medida de CCL asignada a cada alumno para los dife- 
rentes textos mediante el índice kappa, siendo en todos los casos adecuado $(p<.01)$.

Siguiendo la línea de los mencionados autores y otros que clasifican en altos y bajos en comprensión lectora (Dermitzaki \& cols., 2008; Mazzitelli \& col., 2007) se evalúa las siguientes categorías:

1- Lectores hábiles: Hace referencia al alumnado que ha realizado correctamente tanto la evaluación de la comprensión como la regulación.

2- Lectores no hábiles: alumnado que no ha realizado correctamente la evaluación de la comprensión y/o la regulación de la comprensión.

Cabe destacar que los textos incluidos en este instrumento, elaborados para la investigación, referían a temáticas desconocidas por los alumnos, buscando evitar así que los conocimientos previos se interpusieran en el CCL.

\section{Procedimiento}

Con los centros educativos se contactó por carta y telefónicamente con la dirección y el departamento de orientación informándoles de los objetivos de la investigación. Una vez confirmado el interés del centro por participar, se informó por carta a las familias y se les solicitó el consentimiento informado, asegurándoles el anonimato. Por último, se informó al alumnado, se garantizó el anonimato y se les solicitó la colaboración voluntaria. Con el fin de garantizar que los textos fueran adecuados a la edad del alumnado se contó con la opinión del profesorado del centro educativo. De esta forma, se aseguró que, la información utilizada, a la par de encontrarse acorde al momento evolutivo, se refería a temáticas desconocidas por el alumnado.

Los cuestionarios fueron completados de forma colectiva por los estudiantes, en su misma aula, en horario de tutoría. La evaluación fue realizada por los miembros del equipo de investigación EPEDIG, entre los que se encontraba la primera y segunda autora. El tiempo aproximado de aplicación fue de 50 minutos.

Las instrucciones en el caso del cuestionario CEA eran las recomendadas por los autores de dicho instrumento, donde se recalca que el objetivo era conocer qué métodos emplean los estudiantes en relación a sus estudios. En el caso del CCL se informó al alumnado de que la finalidad de la prueba era evaluar los textos para su posible incorporación a libros de asignaturas de ciencias para futuros estudiantes. Por tanto, no eran actividades evaluables para sus calificaciones.

\section{Análisis de datos}

Para realizar los análisis estadísticos se utilizó el programa estadístico IBM SPSS 21.Se han utilizado pruebas paramétricas basando esta decisión en las recomendaciones realizadas por Cordero, Crespo, y Pedraza (2013) al señalar las ventajas de las pruebas paramétricas asociadas a la posibilidad de realizar un análisis más sensible de los resultados, así como el análisis de la eta cuadrado, incluyendo todas las variables explicativas de los resultados en un único análisis en tamaños muestrales medios.

Con el objetivo de estudiar las diferencias entre el grupo de lectores hábiles y no hábiles, se utilizó un ANOVA. Se tuvieron en cuenta los valores de eta cuadrado para describir la proporción de variabilidad de la variable dependiente atribuible a la variable independiente, es decir, la variable lector hábil.

Para establecer las relaciones entre las variables estudiadas, control de la comprensión lectora, estrategias de aprendizaje y el rendimiento académico, se obtuvieron las correlaciones de Pearson, cuyas magnitudes fueron debidamente contrastadas. Por último, con la finalidad de obtener datos sobre la capacidad predictiva de la variable control de la comprensión lectora en el rendimiento académico, se realizó una regresión logística bivariada, donde se calculó la odd ratio (OR).

\section{Resultados}

En la tabla 1 se presenta el ANOVA realizado para comprobar las diferencias entre los grupos lectores hábiles/ no hábiles. Como se puede apreciar existen diferencias entre los grupos en todas las variables, y son diferencias estadísticamente significativas en relación a las siguientes escalas: sensibilización (en concreto control emocional), elaboración (en concreto, selección), personalización (pensamiento crítico y creativo y recuperación) y metacognición. También en las calificaciones de Lengua castellana y Matemáticas y control de la comprensión lectora. En todos los casos las medias son superiores en el grupo de lectores hábiles. Los valores de eta cuadrado giran en torno a 0 en las variables del cuestionario CEA. En el caso de control de la comprensión lectora el valor es de .80, y en las calificaciones medias de Lengua castellana y Matemáticas, los valores giran en torno a 20 .

En la tabla 2 se muestran las correlaciones entre las variables estudiadas. Se puede observar que existen correlaciones positivas entre el control de la comprensión lectora y las calificaciones, así como con las escalas de sensibilización, elaboración, personalización y metacognición. Sin embargo, no existen correlaciones estadísticamente significativas con las subescalas de motivación, actitud, organización y regulación. Las correlaciones entre la calificación de Matemáticas y las variables del cuestionario CEA han resultado positivas y significativas con la escala de sensibilización (excepto en la subescala de actitud), la escala de elaboración (solo en la subescala de selección), y con la escala de metacognición (solo en el caso de la subescala de planificación). No se observan correlaciones con la escala de personalización, excepto en la subescala de 
Tabla 1. Medias, desviaciones típicas, y valores de F, p y eta cuadrado de las variables de estudio en el grupo lectores hábiles y el grupo lectores no hábiles.

\begin{tabular}{|c|c|c|c|c|c|c|c|c|}
\hline Variables & & Grupo & $\begin{array}{c}\mathrm{N}^{\circ} \\
\text { casos }\end{array}$ & Media & $\begin{array}{c}\text { Desviación } \\
\text { típica }\end{array}$ & $F$ & $\mathrm{p}$ & $\begin{array}{c}\text { Eta } \\
\text { cuadrado }\end{array}$ \\
\hline Prueba textos & $\mathrm{CCL}$ & $\begin{array}{c}\text { Hábiles } \\
\text { No hábiles }\end{array}$ & $\begin{array}{l}55 \\
63\end{array}$ & $\begin{array}{c}20,45 \\
3,9\end{array}$ & $\begin{array}{c}4,1 \\
4\end{array}$ & 476,71 & $.000^{* *}$ & .80 \\
\hline $\begin{array}{l}\text { Cuestionario } \\
\text { CEA }\end{array}$ & & & & & & & & \\
\hline \multirow[t]{2}{*}{ Sensibilización } & Escala & $\begin{array}{c}\text { Hábiles } \\
\text { No hábiles }\end{array}$ & $\begin{array}{l}55 \\
63\end{array}$ & $\begin{array}{l}74,75 \\
70,65\end{array}$ & $\begin{array}{c}9,77 \\
11,09\end{array}$ & 4,46 & $.037^{*}$ & .037 \\
\hline & $\begin{array}{c}\text { Control } \\
\text { emocional }\end{array}$ & $\begin{array}{c}\text { Hábiles } \\
\text { No hábiles }\end{array}$ & $\begin{array}{l}55 \\
63\end{array}$ & $\begin{array}{l}17,95 \\
15,70\end{array}$ & $\begin{array}{l}3,94 \\
4,23\end{array}$ & 8,52 & $.004^{* *}$ & .068 \\
\hline \multirow[t]{2}{*}{ Elaboración } & Escala & $\begin{array}{c}\text { Hábiles } \\
\text { No hábiles }\end{array}$ & $\begin{array}{l}55 \\
63\end{array}$ & $\begin{array}{l}61,94 \\
57,86\end{array}$ & $\begin{array}{c}9,71 \\
12,02\end{array}$ & 3,95 & $.049^{*}$ & .034 \\
\hline & Selección & $\begin{array}{c}\text { Hábiles } \\
\text { No hábiles }\end{array}$ & $\begin{array}{l}55 \\
63\end{array}$ & $\begin{array}{l}15,04 \\
13,22\end{array}$ & $\begin{array}{l}2,55 \\
3,02\end{array}$ & 12,17 & $.001^{* *}$ & .095 \\
\hline \multirow[t]{3}{*}{ Personalización } & Escala & $\begin{array}{c}\text { Hábiles } \\
\text { No hábiles }\end{array}$ & $\begin{array}{l}55 \\
63\end{array}$ & $\begin{array}{l}75,25 \\
69,48\end{array}$ & $\begin{array}{l}12,01 \\
12,91\end{array}$ & 6,21 & $.014^{*}$ & .051 \\
\hline & $\begin{array}{l}\text { Pensamiento } \\
\text { crítico y creativo }\end{array}$ & $\begin{array}{c}\text { Hábiles } \\
\text { No hábiles }\end{array}$ & $\begin{array}{l}55 \\
63\end{array}$ & $\begin{array}{l}37,62 \\
34,85\end{array}$ & $\begin{array}{l}6,31 \\
5,82\end{array}$ & 6,06 & $.015^{*}$ & .050 \\
\hline & Recuperación & $\begin{array}{c}\text { Hábiles } \\
\text { No hábiles }\end{array}$ & $\begin{array}{l}55 \\
63\end{array}$ & $\begin{array}{l}14,47 \\
13,05\end{array}$ & $\begin{array}{c}3,12 \\
3,4\end{array}$ & 5,55 & $.014^{\star}$ & .046 \\
\hline \multirow[t]{2}{*}{ Metacognición } & Escala & $\begin{array}{c}\text { Hábiles } \\
\text { No hábiles }\end{array}$ & $\begin{array}{l}55 \\
63\end{array}$ & $\begin{array}{l}38.73 \\
36.06\end{array}$ & $\begin{array}{l}6,16 \\
6,25\end{array}$ & 5,39 & $.022^{*}$ & .044 \\
\hline & Planificación & $\begin{array}{c}\text { Hábiles } \\
\text { No hábiles }\end{array}$ & $\begin{array}{l}55 \\
63\end{array}$ & $\begin{array}{c}23.64 \\
21.9\end{array}$ & $\begin{array}{l}4,51 \\
5,23\end{array}$ & 3,64 & $.014^{\star}$ & .030 \\
\hline \multicolumn{9}{|l|}{ Calificación } \\
\hline & $\begin{array}{l}\text { Calificación } \\
\text { lengua }\end{array}$ & $\begin{array}{c}\text { Hábiles } \\
\text { No hábiles }\end{array}$ & $\begin{array}{l}55 \\
63\end{array}$ & $\begin{array}{c}6.8 \\
5.07\end{array}$ & $\begin{array}{l}1,35 \\
1,78\end{array}$ & 34,50 & $.000^{* *}$ & .22 \\
\hline & $\begin{array}{l}\text { Calificación } \\
\text { matemáticas }\end{array}$ & $\begin{array}{c}\text { Hábiles } \\
\text { No hábiles }\end{array}$ & $\begin{array}{l}55 \\
63\end{array}$ & $\begin{array}{l}6.63 \\
4.85\end{array}$ & $\begin{array}{l}1,78 \\
1,73\end{array}$ & 25,19 & $.000^{* *}$ & .20 \\
\hline
\end{tabular}

Prueba: ANOVA

${ }^{* *} p<.01 \quad{ }^{*} p<.05$

pensamiento crítico y creativo. Respecto a las correlaciones entre la calificación de Lengua castellana y las variables del cuestionario CEA son estadísticamente positivas en el caso de las siguientes escalas: escala de sensibilización (excepto en la subescala de actitud), escala de elaboración (excepto en organización), escala de personalización (excepto transferencia) y con la escala de metacognición (solo con la subescala de planificación).

Con la finalidad de valorar la idoneidad del control de la comprensión lectora como variable predictora, se ha rea- lizado la prueba de regresión logística bivariada entre CCL (como variable dependiente) y calificaciones (como covariada). Para realizar esta prueba se ha recodificado la variable CCL en una variable dicotómica (altos y bajos). El criterio para estar en un grupo o en otro viene dado por la media + $1 / 2 \mathrm{dt}$, es decir, mayor a 16 , se supone en el grupo de CC alto. De esta forma, los resultados indican que la probabilidad es 2.1 (IC 95\%= 1.5 - 2.8) para Lengua castellana y 1.3 (IC $95 \%=1.36-2.4$ ) para Matemáticas. 
Tabla 2. Correlaciones de Pearson entre las variables de estudio.

\begin{tabular}{|c|c|c|c|c|}
\hline Variables & & $\begin{array}{c}\text { Control comprensión } \\
\text { lectora }\end{array}$ & $\begin{array}{l}\text { Media calificaciones } \\
\text { matemáticas }\end{array}$ & $\begin{array}{c}\text { Media calificaciones } \\
\text { lengua }\end{array}$ \\
\hline Prueba de textos & $\begin{array}{c}\text { Control comprensión } \\
\text { lectora }\end{array}$ & & $0,422^{* *}$ & $0,483^{* *}$ \\
\hline \multicolumn{5}{|l|}{ Cuestionario CEA } \\
\hline \multirow[t]{3}{*}{ Escala sensibilización } & Escala & $0,256^{* *}$ & $0,382^{* *}$ & $0,393^{* *}$ \\
\hline & Motivación & - & $0,364^{\star *}$ & $0,410^{\star *}$ \\
\hline & Control emocional & $0,281^{* *}$ & $0,293^{* *}$ & $0,239^{* *}$ \\
\hline \multirow[t]{3}{*}{ Escala elaboración } & Escala & $0,205^{*}$ & $0,204^{*}$ & $0,274^{* *}$ \\
\hline & Elaboración & $0,197^{*}$ & - & $0,207^{*}$ \\
\hline & Selección & $0,309^{* *}$ & $0,267^{* *}$ & $0,310^{* *}$ \\
\hline \multirow{3}{*}{$\begin{array}{c}\text { Escala } \\
\text { personalización }\end{array}$} & Escala & $0,260^{\star *}$ & - & $0,209^{*}$ \\
\hline & $\begin{array}{c}\text { Pensamiento crítico } \\
\text { y creativo }\end{array}$ & $0,301^{* *}$ & $0,227^{\star}$ & $0,188^{*}$ \\
\hline & Recuperación & $0,212^{*}$ & - & - \\
\hline \multirow[t]{2}{*}{ Escala metacognición } & Escala & $0,251^{* *}$ & $0,226^{*}$ & $0,277^{* *}$ \\
\hline & Planificación & $0,224^{*}$ & $0,211^{*}$ & $0,287^{*}$ \\
\hline
\end{tabular}

${ }^{* *} p<.001 \quad{ }^{*} p<.05$

\section{Discusión}

En esta investigación se perseguía relacionar el control de la comprensión lectora con las estrategias de aprendizaje utilizadas y el rendimiento académico en alumnos de secundaria. El control de la comprensión lectora estimado para cada individuo ha permitido clasificar al alumnado como lectores hábiles y lectores no hábiles, y de esta manera indagar más específicamente sobre las estrategias más frecuentemente desplegadas en cada grupo así como su correlación con las calificaciones para las asignaturas de Lengua castellana y Matemáticas.

Los resultados obtenidos muestran que el grupo clasificado como lectores hábiles tiene correlaciones altas y significativas con las calificaciones en las asignaturas de Lengua castellana y Matemáticas. Con respecto a las variables relacionadas con las estrategias de aprendizaje, y aunque los valores de eta son muy bajos, los resultados sugieren la existencia de un perfil diferenciador entre los grupos: los lectores hábiles utilizan más estrategias en comparación con el grupo de lectores no hábiles. Entre dichas estrategias predominan de forma significativa el control emocional, la selección de información relevante, el pensamiento crítico y creativo, la recuperación de la información aprendida y en la planificación y la evaluación de la información como parte del proceso de metacognición.

El control emocional se define como la estrategia para manejar la ansiedad y conseguir un nivel de activación óptimo en la realización de tareas (Beltrán \& cols., 2006). En numerosas investigaciones se subraya la importancia de la ansiedad ante situaciones de evaluación en el ámbito educativo y su repercusión en el rendimiento académico (Serrano, Delgado, \& Escolar, 2010). De hecho, algunos autores destacan la importancia de tener en cuenta las estrategias relacionadas con los aspectos afectivos en el proceso de enseñanza- aprendizaje (Valle \& cols., 2010). De esta forma, se enfatiza la necesidad de investigar sobre la interacción de variables como metacognición y afecto en la autorregulación del proceso de aprendizaje (Efklides, 2011). Sin embargo, en la revisión realizada no se han encontrado estudios que investiguen la posible relación entre el control de la comprensión lectora y la ansiedad lo que sugiere una interesante vía de investigación.

Otras estrategias que predominan en el grupo de lectores hábiles son las siguientes: selección de información relevante, planificación y evaluación de los errores cometidos. Estos resultados son coincidentes con las investigaciones de Dermitzaki y cols. (2008) en un grupo de alumnos denominados altos en comprensión lectora, donde destacaba 
su habilidad para la elección la información principal, la planificación y la reflexión de los errores cometidos. En este sentido, Zimmerman (2008) corrobora que la planificación es una de las estrategias que más capacidad tiene de predecir el éxito en las tareas.

Con respecto al pensamiento crítico y creativo, Beltrán y cols. (2006) entienden que este tipo de estrategia se basa en el pensamiento reflexivo ante la información, búsqueda de razonamientos flexibles para sintetizar en conclusiones. Por pensamiento creativo, aluden los mencionados autores tanto a la flexibilidad mental como a la persistencia ante la tarea, y ambas estrategias son consideradas como metacognitivas. En esta línea, y enfatizando el interés de estas variables, Brincka y Liljenforsb (2013) recuerdan que el entrenamiento en la iniciativa y el mantenimiento de objetivos en la realización de tareas hace que se interioricen las estrategias propias de la metacognición. Los resultados anteriores, confirman los estudios llevados a cabo por algunos autores donde se destaca que el alumnado que se clasifica como buenos lectores utilizan más frecuentemente estrategias de planificación, de control de la comprensión y cognitivas en la realización de tareas del ámbito educativo (Yang, 2012), manifiestan un alto autoconcepto (Madero \& Gómez, 2013) y además destacan en estrategias como auto-activación, persistencia ante dificultades y automotivación (Dermitzaki \& cols., 2008).

Estos datos parecen corroborar lo que algunos estudios sugieren sobre la relación entre las calificaciones y las estrategias de comprensión lectora (González \& cols., 2010). La investigación realizada indica que aquellos alumnos con mejores calificaciones en Lengua castellana y Matemáticas son aquellos que reportan sentirse a gusto en el contexto escolar (escala de actitud), que distinguen las información relevante de la superflua (escala de selección), que se hace preguntas, reflexiona y razona acerca de lo que aprende (escala pensamiento crítico y creativo) y finalmente, son alumnos que dicen planificar sus tareas antes de realizarlas y evaluar sus resultados durante y después de llevarlas a cabo (escala de metacognición). Estos resultados son similares parcialmente con los reflejados en el estudio de Miñano y Castejón (2011), donde se sugiere que las estrategias de aprendizaje tienen un papel mediador en el rendimiento académico, destacando un efecto directo con aptitud verbal, calificaciones en Lengua castellana y con la aptitud matemática y calificaciones de Matemáticas.

En cuanto a la capacidad predictiva que posee el CCL en relación a un mejor rendimiento académico, se ha obtenido, que el uso de esta estrategia se relaciona con una mayor probabilidad de éxito académico, de altas puntuaciones en ambas asignaturas, tanto en Lengua castellana como en Matemáticas, siendo mayor la capacidad predictiva en la primera que en la segunda. Estos resultados, están en consonancia con otros estudios que señalan a la comprensión lectora como una variable fundamental en el proceso de enseñanza- aprendizaje, siendo uno de los aspectos que pueden explicar el rendimiento académico (González \& cols., 2010).
En conclusión, los resultados señalan que el grupo clasificado como lector hábil presenta más estrategias relacionadas con el control emocional, pensamiento crítico y creativo, la selección y recuperación de la información, y finalmente con la planificación y evaluación de los resultados así mismo, presenta correlaciones altas y significativas, entre las calificaciones obtenidas en Lengua castellana y Matemáticas. Por último, el control de la comprensión lectora parece ser una variable predictora del rendimiento académico en las asignaturas analizadas.

\section{Consideraciones finales}

Los métodos utilizados para la evaluación de las variables han seguido las indicaciones señaladas por algunos autores sobre la cuestión. Así, Escribano, Elosúa, Gómez-Veiga, y García-Madruga (2013), recomiendan para la evaluación de la comprensión lectora, el uso de preguntas auto- administradas, después de la lectura de los textos. Es conveniente resaltar que las investigaciones sobre estrategias de aprendizaje y la comprensión lectora provienen de líneas de investigación y metodologicas diferentes, en general, las estrategias de aprendizaje desde una metodología donde predomina el uso del autoinforme; y la comprensión lectora, desde una metodología de generación de preguntas tras la lectura de textos. Según algunos autores, estas diferencias podrían dificultar el estudio de las estrategias de aprendizaje y su relación con la comprensión lectora, aunque recalcan la importancia de diseñar investigaciones donde se pueda contrastar dicha relación (Cano \& cols., 2014).

Es necesario advertir algunas limitaciones de esta investigación que deben tenerse en cuenta, con respecto a la muestra: no ha sido seleccionada al azar y el tamaño es relativamente pequeño, siendo convenientemejorar dichos aspectos en próximos estudios. Asimismo, una de las críticas más frecuente cuando se evalúa el rendimiento académico a través de las calificaciones es que dichas evaluaciones son institucionales, no suelen reflejar un aprendizaje profundo y significativo, y además este aprendizaje no siempre se corresponde con niveles óptimos de rendimiento (Navas, Sampascual, \& Santed, 2003). Por otro lado, es importante destacar las limitaciones intrínsecas que tiene el uso de instrumentos de auto-informe, que aunque contando con las pruebas psicométricas adecuadas, siempre cabe la posibilidad de que las respuestas estén guiadas por un criterio de deseabilidad o conveniencia social.

Se propone como investigaciones futuras la creación de modelos estructurales para poner a prueba estos resultados y así conocer la relación entre el control de la comprensión y las estrategias de aprendizaje que favorezcan la comprensión y su capacidad predictiva en relación al rendimiento académico. Tal y como ya mencionaba Printich (2003) es necesario conocer las variables que influyen en el proceso de activación, adquisición y desarrollo del conocimiento. También es importante tener en cuenta que la adquisición de competencias lectoras redunda en la propia satisfacción del 
estudiante (Long, Ibrahim, \&Kowang, 2014). Los resultados obtenidos en este estudio tienen importantes implicaciones psicoeducativas ya que justifican la necesidad de introducir métodos de enseñanza-aprendizaje que transfieran al alumnado estrategias específicas para mejorar los siguientes aspectos: comprensión lectora, manejar la ansiedad ante situaciones de evaluación, creación y mantenimiento de objetivos académicos, flexibilidad mental y persistencia, así como la planificación. Dichas estrategias redundaría en el proceso de aprendizaje y por tanto, en los resultados académicos. Por último, reseñar que la investigación en este ámbito es fundamental para guiar, introducir y afianzar los cambios que son esenciales en el sistema educativo (Gundy \& Berger, 2015; Bernacky \& cols., 2015; Boudreaux, 2016; Turkyilmaz, 2016).

\section{Referencias}

Azevedo, R. \& Aleven, V. (Orgs.). (2013). International handbook of metacognition and learning technologies. Amsterdam, The Netherlands: Springer.

Anastasious, D. \& Griva, E. (2009).Awareness of reading strategy use and reading comprehension among poor and good readers. Elementary Education Online, 8, 283-297.

Beltrán, J. A., Pérez, J. A., \& Ortega, M. I. (2006). Cuestionario de Estrategias de Aprendizaje (CEA). Madrid: TEA Ediciones.

Bernacky, M. L, Nokes- Malach, T. J.\& Aleven, V. (2015).Examining self- efficacy during learning variability and relations to behavior, performance and learning. Metacognition learning, 10, 99-117.

Boudreaux, M.K. (2016). Survey of developmental students'print and online metacognitive reading.Educational Research Quarterly, 39, 3-22.

Brincka, I., \& Liljenforsb, R. (2013). The Developmental Origin of Metacognition. Infant and Child Infant and Child Development, 22, 85-101.

Cano, F., García, A., Justicia, F., \& García-Berbén, A.B. (2014). Enfoques de aprendizaje y comprensión lectora: el papel de las preguntas de los estudiantes y del conocimiento previo. Revista de Psicodidáctica, 19(2), 247-265.

Cordero, J.M., Crespo, E., \& Pedraja, F. (2013). Rendimiento educativo y determinantes según PISA: Una revisión de la literatura en España. Revista de Educación, 362, 273-297.

Dignath, C. \& Büttner, G. (2008). Components of fostering selfregulated learning among students. A meta-analysis on intervention studies at primary and secondary school level. Metacognition and Learning, 3, 231-264.

Dermitzaki, I., Andreou, G., \& Paraskeva, V. (2008). High and low
Reading comprehension Achievers' Strategic Behaviors and their relation to performance in a reading comprehension situation. Reading Psychology, 29(6), 471-492.

Efklides, A. (2011). Interactions of Metacognition with Motivation and Affect in Self-Regulated Learning: The MASRL Model. Educational Psychologist, 46(1), 6-25.

Escribano, C. L., Elosúa, M. R., Gómez-Veiga, I., \& García-Madruga, J. A. (2013). A predictive study of reading comprehension in thirdgrade Spanish students. Psicothema, 25(2), 199-205.

García-Arista, E., Otero, J., Caldeira, M.H., Prata-Pina, E., Patricio, A., \& Cardoso, E. (2006). El control de la comprensión y el rendimiento académico en ciencias de los alumnos españoles y portugueses. En: J. Díaz de Bustamante \& M. P. Jiménez Aleixandre (Orgs.). Perspectivas sobre a aprendizaxe das Ciencias e das Matemáticas. Estudios en honor ao profesor Eugenio GarcíaRodeja Fernández (pp. 83-95). Santiago de Compostela: Servicio de Publicaciones de La Universidad de Santiago.

González, M.J., Barba, M.J., \& González, A. (2010). La comprensión lectora en secundaria. Revista Iberoamericana de Educación, 53(6), 1-11. Recuperado: 07 jun 2015. Disponível: http://www. rieoei.org/expe/3225Gonzalez.pdf

González-Pienda, J.A., Fernández, E., Bernardo, A., Núñez, J.C. \& Rosário, P. (2014). Assessment of a self-regulated learning intervention. The Spanish Journal of Psychology, 17, 1-9.

Gundy, M. S. \& Berger, M. J. (2015).Towards a model supporting educational change. International.Journal of Information and Education Technology, 6(3), 232-236.

Gutiérrez-Braojo C., Rodríguez, S., \& Salmerón-Vílchez, P. (2014). How can reading comprehension strategies and recall be improved in elementary school students? Estudios sobre Educación, 26, 9-31.

Horner, S. L., \& Shwery, C. S. (2002). Becoming an engaged selfregulated reader. Theory into Practice, 41, 102-109.

Instituto Nacional de Evaluación Educativa [INEE] (2013). PISA 2012. Programa para la evaluación internacional de los alumnos. Informe español (vol. I). Madrid: Ministerio de Educación.

Jiménez-Taracido, L., Baridón, D., \& Manzanal, A.I. (2016). Análisis del control de la comprensión lectoral en textos científicos en alumnos de Secundaria. Revista Complutense de Educación, 27(1), 285-307.

Long, C. S., Ibrahim, Z., \&Kowang, T. O. (2014). An analysis on the relationship between lecturers' competencies and students' satisfaction. International Education Studies, 7(1), 37-46.

Madero, I. P. \& Gómez, L. F. (2013). El proceso de comprensión lectora en alumnos de tercero de secundaria. Revista Mexicana de 
Investigación Educativa, 18(56), 113-139. Recuperado: 5 mai 2015. Disponível: http://www.redalyc.org/articulo.oa?id=14025581006

Mazzitelli, C.A., Maturano, C.I., \& Macías A. (2007). Estrategias de monitoreo de la comprensión de textos de ciencias con dificultades. Enseñanza de las Ciencias, 25(2), 217-228.

Mezzalira, M.A. \& Boruchovitch, E. (2014). Motivación para leer y comprensión lectora en estudiantes brasileños. Educatio siglo XXI, 32 (2), 119-138.

Miñano, P. \& Castejón, J.L. (2011). Variables cognitivas y motivacionales en el rendimiento académico en Lengua Castellana y Matemáticas: un modelo estructural. Revista de Psicodidáctica, 16(2), 203-230.

Navas, L., Sampascual, G., \& Santed, M. A. (2003). Predicción de las calificaciones de los estudiantes: La capacidad explicativa de la inteligencia general y de la motivación. Revista de Psicología General y Aplicada, 56(2), 225-237.

Otero, J. \& Campanario, J.M. (1990). Comprehension evaluation and regulation in learning from science texts. Journal of Research in ScienceTeaching, 27(5), 447-460

Otero, J., Campanario, J. M., \& Hopkins, K. (1992). The relationship between academic achievement and metacognitive comprehension monitoring ability of Spanish Secondary School Students.Educational and Psychological Measurement, 52, 419430.

Pintrich, P. R. (2003). A motivational science perspective on the role of student motivation in learning and teaching contexts. Journal of Educational Psychology, 95(4), 667-686.

Ramírez P. P., Rossel R. P., \& Nazar C. G. (2015). Reading comprehension and metacognition: Analysis of reading activities into two textbooks of seventh year for the course Language and Communication. Estudios pedagógicos, 41(2), 213-231.

Serrano, I., Delgado, J., \& Escolar, M. C. (2010). Eficacia diferencial de estrategias de afrontamiento en la reducción de la ansiedad ante los exámenes en función del tipo de variables principalmente afectada. Ansiedad y Estrés, 16(2-3), 109-126.

Sitzmann, T. \& Ely, K. (2011). A meta-analysis of self-regulated learning in work-related training and educational attainment: What we know and where we need to go. PsychologicalBulletin, 137(3), 421-442.
Solano, P., González-Pienda, J.A., González-Pumariega, S. \& Núñez, J. C. (2004). Autorregulación del aprendizaje a partir de textos. Revista galego-portuguesa de psicoloxia e educación, 9(11), 111128.

Taylor, T.L. \& Hamm, J.P. (2016).Selection for encoding: No evidence of greater attentional capture following forget then remember instrutions. Attention, Perception and Psychophysics, 78(1), 168186.

Tausch, C. (2012). A syntax-based reading intervention for English as second-language learner. Tese de doutorado. Louisiana State University, Louisiana, United States of America. Recuperado: 3 mar 2015. Disponível: http://etd.Isu.edu/docs/available/etd05272012-174438/unrestricted/Tausch_dissertation.pdf

Turkyilmaz, M. (2016). The relationship between reading attitude, metacognitive awareness of reading strategies, personality and self- regulation: A study of modeling. Education, 136(1), 11-18.

Valle, A., Rodríguez, S., Núñez, J. C., Cabanach, R. G., GonzálezPienda, J. A., \& Rosario, P. (2010). Motivación y aprendizaje autorregulado. Interamerican Journal of Psychology, 44(1), 86-97.

Yang, K. L. (2012).Structure of cognitive and metacognitive reading strategy use for reading comprehension of geometry proof. Educational Studies in Mathematics, 80, 307-326.

Young, J. \& Fry, J. (2008).Metacognitive awareness and academic achievement in college students. Journal of the Schoolarship of Teaching and Learning, 8(2), 1-10.

Zimmerman, B. J. (2008). Goal setting: A key proactive source of academic self-regulation. Em D. H. Schunk \& B. J. Zimmerman (Orgs.), Motivation and self-regulated learning. Theory, research and applications (pp. 267-295). New York: Lawrence Erlbaum Associates.

Zimmerman, B. J. (2011). Motivational sources and outcomes of selfregulated learning and performance. Em B. J. Zimmerman \& D. $\mathrm{H}$. Schunk (Orgs.), Handbook of self-regulation of learning and performance (pp. 49-64). New York: Routledge. 


\section{Sobre as autoras}

Natalia Solano Pinto (natalia.solano@uclm.es)

Licenciada y Doctora en Psicología. Asesora externa del grupo de investigación EPEDIG (Universidad Internacional de la Rioja).Universidad Castilla La Mancha. Departamento de Psicología. Área de Psicología Evolutiva y de la Educación. Facultad de Educación. Campus de Toledo. España.

Ana Isabel Manzanal Martínez (ana.manzanal@unir.net)

Licenciada y Doctora en Ciencias Biológicas, y Licenciada en Ciencias Ambientales. Profesora asociada. Universidad Internacional de La Rioja (UNIR). Departamento de Ciencias Experimentales y su Didáctica. Facultad de Educación. Investigadora del grupo EPEDIG (UNIR).

Lourdes Jiménez-Taracido (lourdes.jimenez@unir.net)

Licenciada Ciencias del Mar y Doctora en Tecnologías del Medio Ambiente. Profesora asociada. Universidad Internacional de La Rioja (UNIR).

Área de Postgrado. Facultad de Educación. Investigadora del grupo INCISO (UNIR).

Esta actividad ha sido parcialmente financiada por UNIR Research (http://research.unir.net), Universidad Internacional de La Rioja (UNIR, http:// www.unir.net), dentro del Plan Propio de Investigación, Desarrollo e Innovación [2013- 2015], Grupo de Investigación EPEDIG. 